\title{
Unusual Location of a Recurrent Epidermoid Cyst
}

\author{
Oumniya Abouhanine, Aicha Merzem, Vianney Ndayishimiye, Omar Amriss, Hasnaa Belgadir, Nadia Moussali, Naima El Benna \\ Department of Radiology, 20 August 1953 Hospital, University Hospital Center Ibn Rochd, Hassan II University, Casablanca, Morocco
}

\section{Doi: 10.12890/2020_002009 - European Journal of Case Reports in Internal Medicine - ๑ EFIM 2020}

Received: $17 / 09 / 2020$

Accepted: 20/09/2020

Published: 19/11/2020

How to cite this article: Abouhanine O, Merzem A, Ndayishimiye V, Amriss O, Belgadir H, Moussali N, El Benna N. Unusual location of a recurrent epidermoid cyst. EJCRIM 2020;7: doi:10.12890/2020_002009.

Conflicts of Interests: The Authors declare that there are no competing interests.

This article is licensed under a Commons Attribution Non-Commercial 4.0 License

\section{ABSTRACT}

Introduction: Epidermoid cysts of the temporal bone are rare, benign and slow-growing lesions.

Patient and Methods: We report the case of a 69-year-old female patient followed up for a symptomatic intradiploic epidermoid cyst of the temporal scale and left mastoid region, which had been operated on but recurred.

Results: MRI demonstrated a well-limited lesion seen as a hyposignal on T1-weighted images, hypersignal on T2-weighted images, on FLAIR and on diffusion-weighted images not enhanced by gadolinium. The tumour was compressive, and bone lysis was seen on CT.

Conclusion: Epidermoid cysts of the temporal bone are rare, benign lesions whose diagnosis is based on fluid signals seen on MRI but absent on FLAIR sequences.

\section{LEARNING POINTS}

- Epidermoid cysts of the temporal bone are rare, benign and slow-growing lesions.

- Larger cysts are easier to identify.

- Diagnosis is based on the presence of a fluid signal on all MRI sequences except for FLAIR imaging, and on confirmation by pathological examination.

\section{KEYWORDS}

Epidermoid, cyst, intradiploic, temporal bone, recurrent

\section{INTRODUCTION}

Epidermoid cysts of the temporal bone are extremely uncommon. They are rare, benign extra-axial tumours of the skull which develop in the diploe. They are slow growing, can reach an enormous size, and cause mild neurological signs.

This report describes the CT and MRI imaging findings of a symptomatic, recurrent intradiploic epidermoid cyst of the scale of the temporal bone and of the mastoid region.

\section{CASE DESCRIPTION}

We report the case of a 69-year-old patient followed up for an operated and recurrent intradiploic epidermoid cyst of the temporal scale and the left mastoid region. Symptoms began in 2010 with headaches and dizziness not improved by medical treatment, which prompted an ENT consultation. A radiological assessment was requested and identified a tumour in the left temporal region which was operated on and identified as an epidermoid cyst. This lesion reappeared in 2012 and in 2015, and was operated on again. The most recent recurrence was at the end of 2019, motivating another consultation. 
MRI demonstrated an oval formation of intradiploic appearance of the scale of the temporal bone and of the left mastoid region, which was well limited and had a thin wall. It measured $65 \times 38 \times 69 \mathrm{~mm}$ and pressed on the temporal lobe and the left cerebellar hemisphere. There was no cochleo-vestibular bundle abnormality (Figs. 1 and 2)

A CT scan showed this formation was hypodense and responsible for bone lysis of the mastoid and the internal table of the temporal bone (Fig. 3).

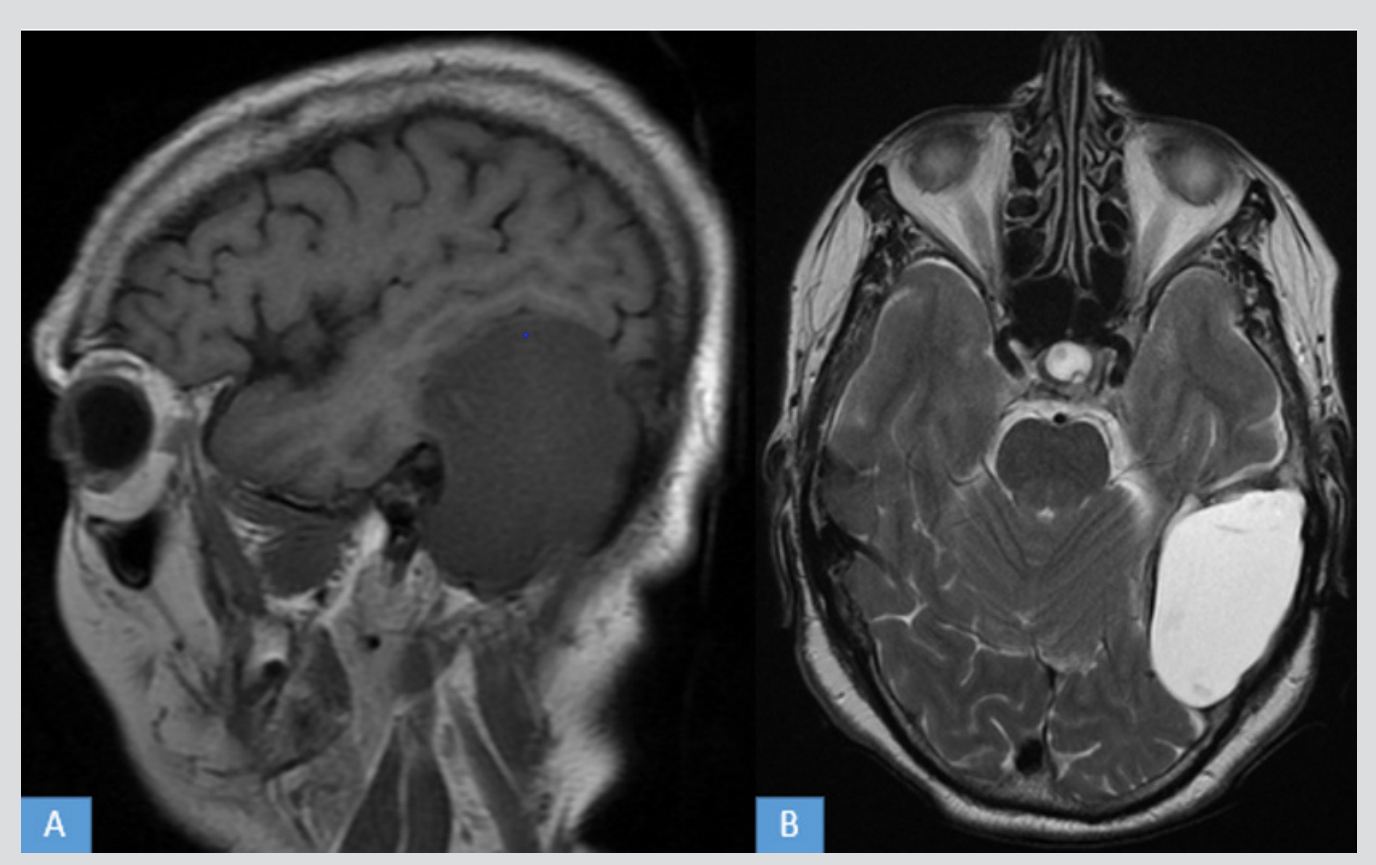

Figure 1. Cerebral MRI: (A) sagittal T1 sequence, (B) axial T2 sequence. formation of the left temporal and mastoid region in T1 hyposignal, T2 hypersignal with thin and irregular wall, site of a few hypointense areas on T2-weighted imaging

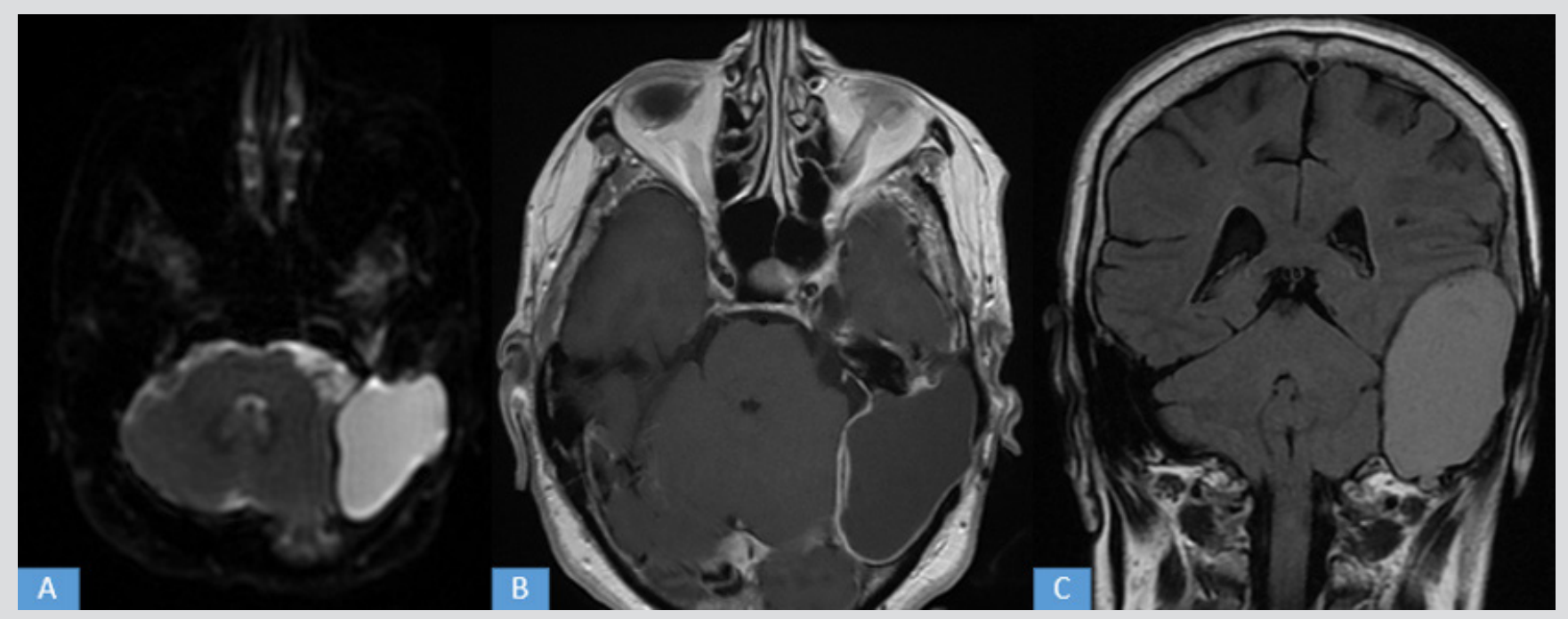

Figure 2. Cerebral MRI: (A) axial diffusion, (B) coronal (FLAIR), and (C) axial T1 sequence after gadolinium injection. This formation is in hypersignal diffusion and FLAIR not enhanced by gadolinium

\section{DISCUSSION}

Epidermoid cysts are congenital lesions of ectodermal origin, which result from incomplete cleavage between the neuroectoderm and the ectoderm of the skin during closure of the neural tube towards the 4th week of embryonic life, which leads to the inclusion of ectodermal cells. An epidermoid cyst has a wall with an outer fibrous capsule and an epidermal-like epithelium internally. 


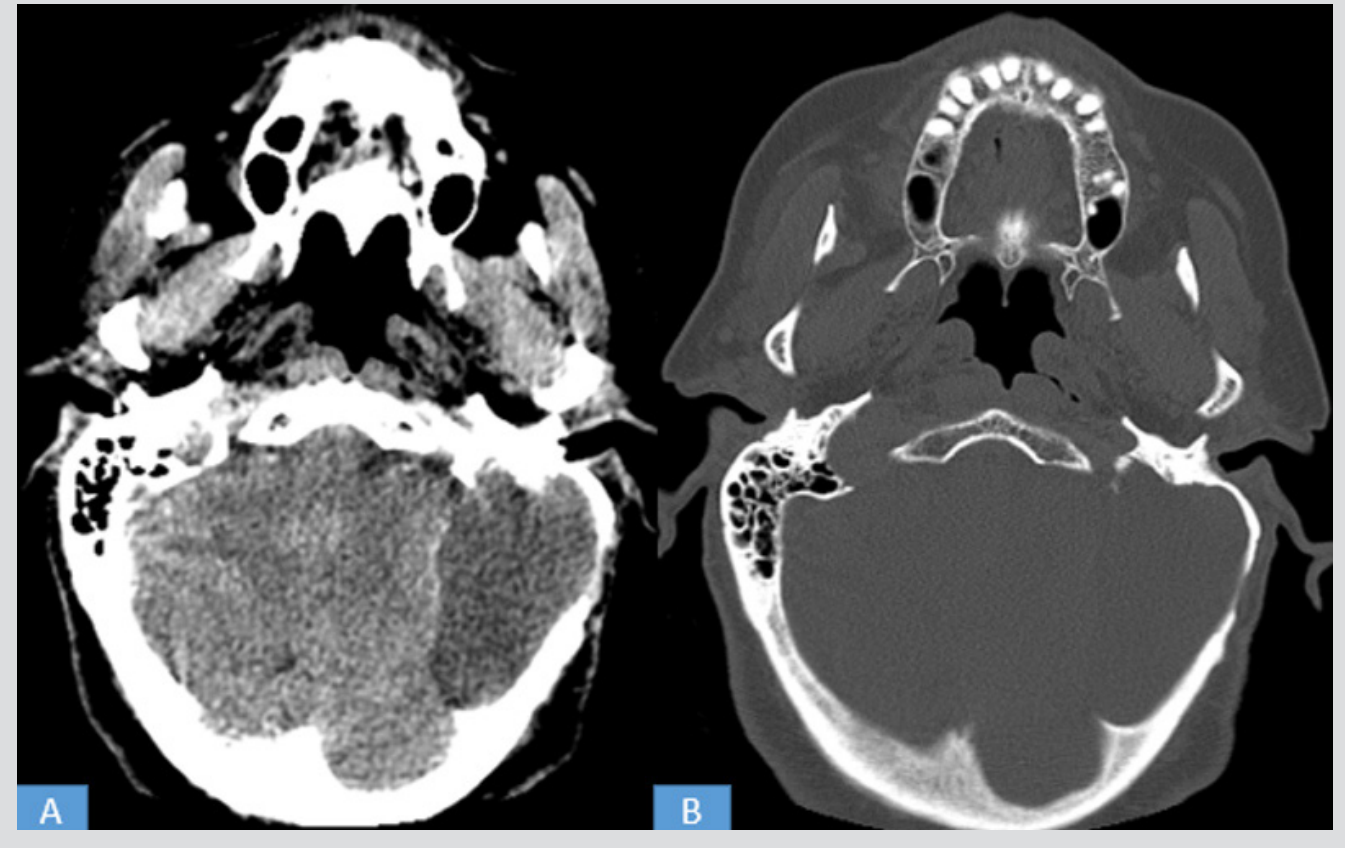

Figure 3. Cerebral CT scan: (A) axial section parenchymal window and (B) bone window. This formation is hypointense with an irregular thin-wall. It pushes on the bone cortex and erodes the ipsilateral mastoid cells.

Epidermoid cysts account for approximately $1 \%$ of intracranial tumours and $5 \%$ of cerebellar angle tumours. The most frequent intradural locations ( $90 \%$ of cases) of epidermoid cysts are the pontocerebellar angle ( $50 \%$ of cases), suprasellar cistern, temporal fossa, quadrigeminal cistern and large cistern. Location at the hemispherical or intraventricular convexity level (V4, choroidal fissure and lateral ventricle) is rare. Extradural epidermoid cysts ( $10 \%$ of cases) are located intradiploic at the level of the cranial vault, parietal and occipital, at the level of the large wing of the sphenoid, and at the level of the petrous apex ${ }^{[1]}$. Localization in the mastoid region was described in two patients by Syed et al. ${ }^{[2]}$.

Epidermoid cysts are most commonly seen in the third and fourth decades of life ${ }^{[3]}$, and gradually increase in size due to the accumulation of epidermal desquamation products in the form of keratin and cholesterol crystals. They are mainly discovered incidentally on $x$-ray performed for other purposes.

The clinical presentation depends on the site of origin and the size of the tumour and includes progressive headache and tinnitus without neurological deficits, a picture of intracranial hypertension, and focal neurological signs in the event of large tumours ${ }^{[4]}$.

On standard radiography, intraosseous epidermoid cysts are generally responsible for well-circumscribed osteolysis, with clear and regular surroundings, sometimes bounded by peripheral sclerosis ${ }^{[5]}$.

On CT scans, epidermoid cysts typically appear in the form of an iso or hypodense, heterogeneous, extra or intra-axial mass, with irregular contours, with no enhancement after injection of contrast product, and without perilesional oedema. Rarely, cysts are hyperdense due to calcification, haemorrhage, high protein content or degeneration ${ }^{[6]}$.

The MRI appearance of epidermoid cysts is identical regardless of their location. They are isointense on T1-weighted images and hyperintense on T2-weighted images, with clear but irregular limits, without perilesional oedema or contrast enhancement. The signal is often inhomogeneous on the FLAIR sequence; it can be variable in intensity depending on the protein content of the tumour. Atypical forms have been reported, with a spontaneously hyperintense mass on T1-weighted images and a hypointense mass on T2-weighted images, probably due to the presence of calcifications, and a high protein content with a heterogeneous appearance on the FLAIR sequence, increased in the signal on the diffusion sequence, especially with the hyperintense and heterogeneous aspect in sequence CISS-3D ${ }^{[7]}$.

Epidermoid cysts are benign lesions, although rare cases of malignant transformation into squamous cell carcinoma have been reported. Cysts that have undergone multiple operations and repeated infections are probably at a greater risk of malignant degeneration and the prognosis in these cases is very poor ${ }^{[4]}$.

The differential diagnosis of such temporal bone lesion includes cholesterol granuloma, dermoid cyst, hydatid cyst, haemangioma, arachnoid cyst, eosinophilic granuloma, meningioma, a repairing giant cell granuloma and an aneurysmal bone cyst ${ }^{[2]}$. 


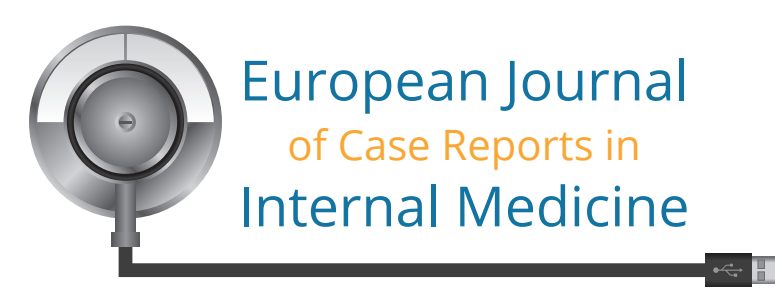

\section{CONCLUSION}

Epidermoid cysts of the temporal bone are rare, benign and slow-growing lesions. Diagnosis is based on MRI and CT images which demonstrate a classic liquid hyposignal on all sequences except for FLAIR.

\section{REFERENCES}

1. Dietemann J-L. Neuro-imagerie diagnostique. Issy-les-Moulineaux: Elsevier Masson; 2012

Syed MI, Plodpai Y, Khoo SG, Rutka JA. Primary epidermoid cysts of the mastoid: clinical and treatment implications. Eur Arch Otorhinolaryngol 2016:273(4):1055-1059.

3. Twede JV, Patterson MC, Anderson ML. Intraosseous epidermoid cyst of the skull: case study and radiological imaging considerations. Dermatol Online J 2018;24(7):13030/ qt5712f7zb.

4. Bikmaz K, Cosar M, Bek S, Gokduman CA, Arslan M, Iplikcioglu AC. Intradiploic epidermoid cysts of the skull: a report of four cases. Clin Neurol Neurosurg 2005;107(3):262-267.

5. Lambert A, Larousserie F, Drapé J-L. Tumeurs osseuses bénignes: kystes épidermoïdes. EMC - Radiologie et Imagerie Médicale - Musculosquelettique - Neurologique - Maxillofaciale 2010;5(4):1-6.

6. Arai A, Sasayama T, Koyama J, Fujita A, Hosoda K, Kohmura E. Epidermoid cyst in Meckel's cave with unusual computed tomography and magnetic resonance imaging findings. Neurol Med Chir (Tokyo) 2010;50(8):701-704.

7. Saqui AE, Aggouri M, Benzagmout M, Chakour K, Faiz ME. Kyste épidermoïde du quatrième ventricule: à propos d'un cas. Pan Afr Med J 2017;26:239. 